\title{
Optimal Coordination of Intermittent Distributed Generation with Probabilistic Power Flow
}

\author{
Haijun Xing ${ }^{\dagger}$, Haozhong Cheng* and Yi Zhang**
}

\begin{abstract}
This paper analyzes multiple active management (AM) techniques of active distribution network (ADN), and proposes an optimal coordination model of intermittent distributed generation (IDG) accommodation considering the timing characteristic of load and IDG. The objective of the model is to maximize the daily amount of IDG accommodation under the uncertainties of IDG and load. Various active management techniques such as IDG curtailment, on-load tap changer (OLTC) tap adjusting, voltage regulator (VR) tap adjusting, shunt capacitors compensation and so on are fully considered. Genetic algorithm and Primal-Dual Interior Point Method (PDIPM) is used for the model solving. Point estimate method is used to simulate the uncertainties. Different scenarios are selected for the IDG accommodation capability investigation under different active management schemes. Finally a modified IEEE 123 case is used to testify the proposed accommodation model, the results show that the active management can largely increase the IDG accommodation and penetration.
\end{abstract}

Keywords: Active distribution network, Active management, Intermittent distributed generation, Point estimate method, Probabilistic power flow

\section{Introduction}

With the economy fast development and urbanization moving forward in China, electricity demand of urban center keeps growing. The traditional centralized mass power generation cannot fulfill the request for clean production and efficient utilization of electricity energy, but brings heavy pressure to the environment. According to IEA (International Energy Agency) statistics, the world's $\mathrm{CO}_{2}$ emissions are over 7 billion tons in 2010. Chinese emissions far surpass those of the other BRICS countries and account for $24 \%$ of global emissions. Generation of electricity and heat was by far the largest producer of $\mathrm{CO}_{2}$ emissions and was responsible for $41 \%$ of world $\mathrm{CO}_{2}$ emissions in 2010 , emissions in the electricity and heat generation sector represent $50 \%$ of Chinese $\mathrm{CO}_{2}$ emissions in $2010[1,2]$. In this background, we can forecast the distributed generation (DG), especially the intermittent distributed generation (IDG) will have a striking future by virtue of environment-friendly characteristic.

DG usually refers to the small-sized independent generation system closed to the users, which includes the micro-turbine generation (MTG), wind turbine generation (WTG), photovoltaic generation (PVG), energy storage,

$\dagger$ Corresponding Author: Key Laboratory of Control of Power Transmission and Conversion, Ministry of Education, Shanghai Jiao Tong University, Shanghai, China. (xinghj601@126.com)

* Key Laboratory of Control of Power Transmission and Conversion, Ministry of Education, Shanghai Jiao Tong University, Shanghai, China. (hzcheng@sjtu.edu.cn)

** Fujian Electric Power Research Institute, Fujian, China. (zhangyiscu@163.com)

Received: April 2, 2015; Accepted: June 5, 2015 etc.

DG, as one of the important way for the intermittent renewable energy (IRE) utilization, has made fast progress these years. The European Union's integration program plans to increase the DG capacity to $30 \%$ of the total installed power generation capacity in 2030, through the power management within the European Union countries.

By the end of 2012, the total RES (Renewable Energy Source) generation capacity in China rises to $313 \mathrm{GW}$, within which the WTG capacity is $60.83 \mathrm{GW}$; the photovoltaic generation (PVG) is $3.28 \mathrm{GW}$ [3]. From 2006 to 2012, the average annual growth rate of dispatchable WTG capacity is $76 \%$. According to the national wind power development planning, the WTG scale will rise to $100 \mathrm{GW}$ in 2015 and $200 \mathrm{GW}$ in 2020[4]. In order to achieve the goal, fine down the interconnect regulation, State Grid Corporation of China (SGCC) finished the revision of the relevant technical specifications for DG interconnection in distribution network, and the management opinion for promoting the DG interconnection in distribution network, etc.

The IDG accommodation in distribution system is connecting dispersedly to the MV (Medium Voltage) and LV (Low Voltage) distribution network [5-7]. Reference [5] determines the optimal accommodation of renewable DG in a way that minimizes the system energy losses based on multi-period AC optimal power. The multi-objective, energy losses and more generation capacity, is also investigated. Reference [7] increases the penetration of IDG through the energy storage. The energy storage facility accommodates the surplus energy from IDG in the 
period of ample supply or lower demand, e.g. the night; supplies back to the users when the IDG output decreases or demand increases, e.g. the peak time in the day.

The rapid expansion of DG brings a series of problems, such as voltage rising of interconnect point, bi-directional power flow, short circuit current rising, etc. Because of the traditional passive management mode, the positive role of DG for improving the distribution system performance cannot be fully excavated. On the contrary, the uncertainties brought by IDG will affect the safe and stable operation of the distribution system. Hence, the distribution system operator (DSO) has to strictly control the capacity and output of interconnected DG. When fault happens, DG is the earliest one to be curtailed.

Aimed at the shortcomings of the passive management mode, the Active Distribution Network (ADN) and Active Management (AM) are proposed to increase the IDG accommodation. Reference [8-11] introduce the research progresses, key technologies and challenges of ADN, summarize the necessity and feasibility to develop the ADN. Reference [12-16] propose to improve the interconnected DG capacity of distribution system through Active Power Flow Management (APFM). Reference [16] evaluates the maximum wind energy exploitation in ADN based on a multi-period optimal power flow (OPF). The AM schemes such as coordinated voltage control, energy curtailment and power factor control are considered. Reference [17] proposes a combined problem formulation for activereactive OPF in distribution network with WTG and battery storage. The proposed approach can reduce the power loss and reactive energy consumption in comparison to the operation strategy where only active OPF is considered. Reference [18] proposes two power flow management (PFM) methodologies, multiple scenarios are presented to demonstrate the algorithms ability of detecting and alleviating thermal overloads. Reference [19] uses the multiple time period dynamic OPF to schedule energy storage and flexible demand for reducing the generator curtailment.

After detailed measurement and assessment of the system operation data, AM can coordinate the operation between DG and other equipment in distribution network, improve DG accommodation, enhance the system reliability and power quality. The distribution network with AM can optimize system operation through regulating the DG output, adjusting the OLTC tap position, reactive power compensation and etc.

This paper are organized as follows: Section 2 explains the ADN and AM, based on a simplified distribution network, different AM schemes are introduced. Section 3 presents the IDG accommodation mathematic model, point estimate for uncertainties and the solving procedure. IEEE 123 case is tested and discussed in Section 4. Finally, in Section 5, some relevant conclusions are summarized.

\section{ADN and AM}

\subsection{ADN}

The traditional distribution network passively accepts the energy from transmission network and distributes to the users. The original intention of distribution network has not included DG. The purpose of the close-looped design and open-looped operation of traditional distribution network is to minimize the interference in the distribution network during daily operation. Only when fault occurs, the interference is permitted, the users of the fault feeder are transferred to the non-fault feeder through the closelooped structure.

From above, the traditional distribution networks are not ready for DG integration, a more active network and capable for large-scale DG integration is necessary. In year 2006, Christian D'ADAMO from the Distribution Systems and Disperse Generation work group (C6) of International council on Large Electric System (CIGRE) created a new task force Development and Operation of Active Distribution Networks (C6.11). From investigation, C6.11 defines the ADN as below [20]:

Active distribution networks have systems in place to control a combination of distributed energy resources (DERs), defined as generators, loads and storage. Distribution system operators have the possibility of

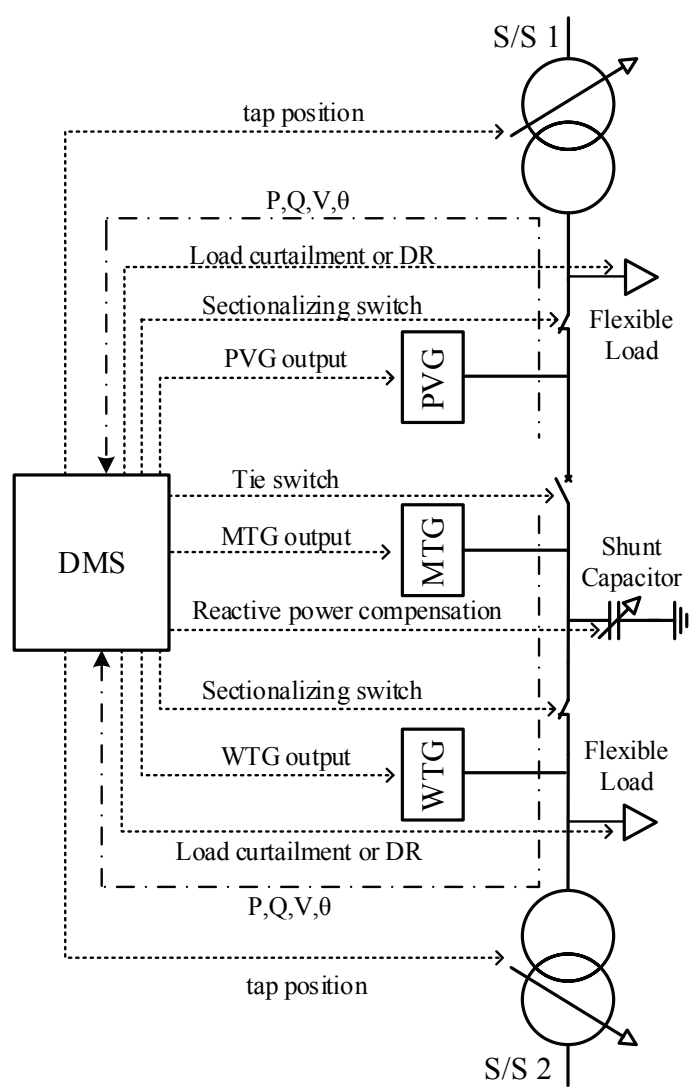

Fig. 1. Operation and Control of ADN 
managing the electricity flows using a flexible network topology. DERs take some degree of responsibility for system support, which will depend on a suitable regulatory environment and connection agreement.

The core of ADN is AM, which depends on the advanced communication and measuring technologies. These technologies can strengthen the monitoring ability of distribution network, control DG with specified operation mode, improve the system visibility and transfer the passive distribution network to an active one.

Fig. 1 shows the simple operation and control diagram of ADN. The dot dash line is the detection line for the total distribution system and sending back to Distribution Management System (DMS). The dotted line stands for the DMS sending orders to the equipment in the distribution network and implementing active management and control.

At present, the global management and optimal control of ADN mainly reflects in power flow optimal management, voltage active control, DG coordinated control, network fast reconfiguration, etc. Through real time monitoring the load data, switch status and DG operation status, the DSO can manage and control the distribution network from an overall situation, obtain a management strategy with all the network constraints satisfied.

\section{$2.2 \mathrm{AM}$}

The AM consists of DG output dispatching and curtailment, reactive power compensation, On-Load Tap Changer (OLTC) tap adjusting, controllable load (demand response), voltage regulator, network reconfiguration, etc. Fig. 2 is a simplified distribution network with DG, OLTC and shunt capacitor.

In Fig. 2, $P_{L}, Q_{L}$ is the active and reactive power of the load; $P_{D G}, Q_{D G}$ is the injected active and reactive power from $\mathrm{DG} ; Q_{C}$ is the injected reactive power from capacitor. The line impedance between bus 1 and 2 is $R+j X$. The voltage of bus 2 can be calculated by the following formula:

$$
U_{1} \cong U_{2}+\left[\left(P_{L}-P_{D G}\right) R+\left(Q_{L}-Q_{D G}-Q_{C}\right) X\right] / U_{2}
$$

Let $Q=Q_{L}-Q_{D G}-Q_{C}, Q$ is the reactive power outputted from bus 2 . The injected intermittent active power on bus 2 can be obtained from (1):

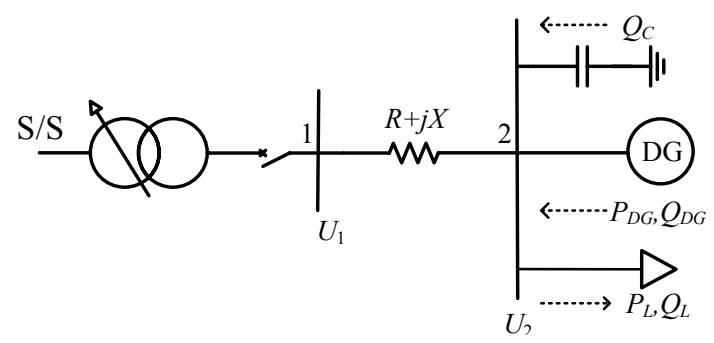

Fig. 2. Simplified Network for AM analysis

$$
P_{D G}=P_{L}+\left[U_{2}\left(U_{2}-U_{1}\right)+Q X\right] / R
$$

The following parts will analyze the different AM schemes of ADN with the purpose to prevent the voltage violation on the DG connecting bus. In normal operation condition, $U_{2}$ should be great that $0.5 U_{1}, U_{2}\left(U_{2}-U_{1}\right)+Q X$ is monotonically increasing. From (2), the voltage of bus 2 will most likely violate with minimum load and maximum intermittent energy output. Assume the voltage upper limit of bus 2 is $U_{2}^{\max }$, we can derive the permitted upper limit of the connected intermittent energy is:

$$
P_{D G}^{\max }=P_{L}^{\min }+\left[U_{2}^{\max }\left(U_{2}^{\max }-U_{1}\right)+Q X\right] / R
$$

where, $P_{L}^{\min }$ is the minimum load occurred during the operation. The effect of different AM schemes to the DG accommodation will be discussed as bellows:

\subsubsection{DG output dispatching}

When the intermittent energy can be curtailed partially, the permitted maximum DG interconnected can be formulated as (4).

$$
P_{D G}^{\max }=P_{D G}^{c u r}+P_{L}+\left[U_{2}^{\max }\left(U_{2}^{\max }-U_{1}\right)+Q X\right] / R
$$

where, $P_{D G}^{c u r}$ is the permitted DG curtailment. More intermittent energy can be accommodated when DG curtailment is permitted in lower power demand time.

\subsection{2 reactive power compensation}

From (2) and $Q=Q_{L}-Q_{D G}-Q_{C}$, the reactive power compensation equipment has to change from injecting reactive power to the system to absorbing reactive power from the system in order to increase the accommodation of DG.

From (2), the effect of the reactive power compensation for the DG accommodation depends on the reactance $X$. The effect of over-head network (the reactance is big, the reactance of over-head line without ground wire is about $0.4 \Omega / \mathrm{km}$ ) is better than the cable network (the reactance is small, the reactance of 3-core cable line is about 0.08 $\Omega / \mathrm{km})$. But, it is important to bear in mind that absorbing reactive power would lead to an increase in system power losses.

\subsubsection{OLTC or VR tap adjusting}

From (2), adjusting the OLTC tap position to make voltage on bus 1 lower can increase the intermittent energy accommodation. The maximum intermittent energy accommodated can be formulated as (5).

$$
P_{D G}^{\max }=P_{L}+\left[U_{2}^{\max }\left(U_{2}^{\max }-U_{1}^{\min }\right)+Q X\right] / R
$$

where $U_{1}^{\min }$ is the permitted lowest voltage on bus 1 . 


\subsection{4 controllable load (demand response)}

From (2), the load shedding surely will decrease the amount of DG that can be accommodated. But as to a complex distribution network with multiple DG, it is hard to give a conclusion. The interconnected DGs change the power flow distribution of the whole network, proper load shedding can prevent the parameters violation of distribution network during operation, thus increase the amount of DG accommodated.

\subsection{5 network reconfiguration}

Network reconfiguration is somewhat like the controllable load. They change the power flow distribution of the whole network, and prevent the violation of distribution network parameters, thus increase the amount of DG accommodation.

\section{The IDG accommodation in ADN}

\subsection{IDG accommodation mathematic model}

The principle of the IDG accommodation considering the timing characteristic is to actively control the equipment in the distribution network according to the load forecasting, WTG and PVG output forecasting, power flow information in every moment, thus minimize the IDG curtailment with all the constraints satisfied. The final purpose is to maximize the amount of WTG and PVG that accommodated within the whole study period. The detailed accommodation model is shown below:

$$
\max f=\sum_{t}\left(\sum_{i=1}^{n} P_{I D G, i}^{t}\right)
$$

where $P_{I D G, i}^{t}$ is the output of the $i$ th IDG at time $t, n$ is the number of installed IDG, including WTG and PVG.

$$
\begin{aligned}
& \text { s.t. } \\
& \qquad \begin{array}{c}
P_{G i}-P_{L i}=U_{i} \sum_{j \in i} U_{j}\left(G_{i j} \cos \delta_{i j}+B_{i j} \sin \delta_{i j}\right) \\
Q_{G i}+Q_{C i}-Q_{L i}=U_{i} \sum_{j \in i} U_{j}\left(G_{i j} \sin \delta_{i j}-B_{i j} \cos \delta_{i j}\right) \\
U_{i}^{\min } \leq U_{i} \leq U_{i}^{\max } \\
S_{i} \leq S_{i}^{\max } \\
P_{I D G, i}^{\min } \leq P_{I D G, i} \leq P_{I D G, i}^{\max } \\
Q_{I D G, i}^{\min } \leq Q_{I D G, i} \leq Q_{I D G, i}^{\max } \\
Q_{C, i}^{\min } \leq Q_{C, i} \leq Q_{C, i}^{\max } \\
\operatorname{Tap}_{k}^{\min } \leq \operatorname{Tap}_{k} \leq \operatorname{Tap}_{k}^{\max } \\
P_{L c u r, i} \leq P_{L c u r, i}^{\max } \\
Q_{I D G, i}=g\left(P_{I D G, i}\right)
\end{array}
\end{aligned}
$$

where $P_{G i}, Q_{G i}$ is the real and reactive power of generators connected on bus $i ; P_{L i}, Q_{L i}$ is the real and reactive power of load connected on bus $i$; $Q_{C i}$ is the reactive power of capacitors connected on bus $i ; U_{i}, U_{j}$ is the voltage amplitude at bus $i$ and $j$ separately; $j \in i$ represents all the buses connected with bus $i ; G_{i j}, B_{i j}, \theta_{i j}$ are separately electric conductance, susceptance and phase angle difference between bus $i$ and $j ;(7)$ is the bus power balance constraint.

Eq. (8) is the bus voltage constraint, each bus voltage magnitude must lie within the permitted range in order to maintain the power quality. $U_{i}, U_{i, \max }, U_{i, \min }$ are separately the bus $i$ voltage and voltage limits; (9) is the branch capacity constraint, each branch apparent power must be less than the maximum permitted capacity. Where $S_{i}$, $S_{i}^{\text {max }}$ are apparent power and maximum permitted capacity of branch $i$.

Eq. (10) is the IDG output constraint, the active and reactive power of each IDG injected to the distribution network must be restricted in a suitable range. $P_{I D G, i}$, $P_{I D G, i}^{\min }, P_{I D G, i}^{\max }$ are $I D G_{i}$ real power output and limits; $Q_{I D G, i}$ $Q_{I D G, i}^{\min }, Q_{I D G, i}^{\max }$ are $I D G_{i}$ reactive power output and limits.

Eq. (11) is the reactive power compensation constraint, $Q_{C i}, Q_{C i}^{\max }, Q_{C i}^{\min }$ are the injected reactive power and limits of bus $i$.

Eq. (12) is the OLTC or VR tap position adjusting range constraint, $\operatorname{Tap}_{k}, \operatorname{Tap}_{k}^{\max }, \operatorname{Tap}_{k}^{\min }$ are the $k$ th tap position and position limits.

Eq. (13) is the load curtailment or demand response constraint, $P_{\text {Lcur }, i}, P_{\text {Lcur }, i}^{\max }$ are the load curtailment and limit of bus $i$.

Eq. (14) is the function of active, reactive power of WTG and PVG, which is aimed at the constant power factor control for IDG.

All the above variables in the constraint equations without limit symbols are the values at time $t$ during the multi-period optimization.

\subsection{Point estimate for probabilistic power flow}

In this paper, the uncertainties of load, wind speed or intensity of illumination are considered. The point estimate method is used to solve the probabilistic OPF. Point estimate method uses deterministic method for solving probabilistic problems with lower computational burden compared with the classic Monte Carlo simulation. Furthermore, point estimate method overcomes the difficulties associated with the lack of perfect knowledge of the probability functions of stochastic variables, since these functions are approximated using only their first few statistical moments [21-23].

Let $p_{l}$ be load, wind speed or intensity of illumination, which is a random variable with probability density function $f_{p l}$. The point estimate method uses two of $p_{l}$ variables, $p_{l, 1}$ and $p_{l, 2}$, which can be calculate with (15) to substitute $p_{l}$ by matching the first three moments of $f_{p l}$. 


$$
p_{l, k}=\mu_{p l}+\xi_{l, k} \times \sigma_{p l} \quad k=1,2
$$

where $\mu_{p l}$ and $\sigma_{p l}$ are the mean and standard deviation of $f_{p l}$.

$$
\xi_{l, k}=\lambda_{l, 3} / 2+(-1)^{(3-k)} \times \sqrt{m+\left(\lambda_{l, 3} / 2\right)^{2}} \quad k=1,2
$$

$\lambda_{l, 3}$ is the coefficient of skewness of $p_{l}$, which can be calculated as follows:

$$
\begin{gathered}
\lambda_{l, 3}=E\left[\left(p_{l}-\mu_{p l}\right)^{3}\right] /\left(\sigma_{p l}\right)^{3} \\
E\left[\left(p_{l}-\mu_{p l}\right)^{3}\right]=\sum_{t=1}^{N}\left(\mu_{p l}-p_{l, t}\right)^{3} \times \operatorname{Prob}\left(p_{l, t}\right)
\end{gathered}
$$

where $N$ is the number of observation of $p_{l}, \operatorname{Prob}\left(p_{l, t}\right)$ is the probability of each observation $p_{l, t}$. The weighting factor $\omega_{l, k}$ of the concentration located at $\left(\mu_{p 1}, \mu_{p 2}, \ldots, p_{l, k} \ldots, \mu_{p \mathrm{~m}}\right)$ can be obtained from (19).

$$
\omega_{l, k}=1 / n \times(-1)^{k} \times\left(\xi_{l, 3}-k\right) / \varsigma_{l}
$$

where $s_{l}=2 \sqrt{m+\left(\lambda_{l, 3} / 2\right)^{2}}$. Once all the concentrations $\left(p_{l, k}, \omega_{l, k}\right)$ are obtained, the OPF model to solve the maximum IDG accommodation $f$ is evaluated at points $\left(\mu_{p 1}\right.$, $\left.\mu_{p 2}, \ldots, p_{l, k} \ldots, \mu_{p \mathrm{~m}}\right)$ yielding $Z(l, k)$, where $Z$ is the vector of output random variables. Then the $j$ th moment of $Z$ can be formulated as follows:

$$
E\left[Z^{j}\right] \cong \sum_{l=1}^{m} \sum_{k=1}^{2} \omega_{l, k} \times[Z(l, k)]^{j}
$$

The standard deviation of $Z$ can be obtained with (21):

$$
\sigma_{Z}=\sqrt{E\left(Z^{2}\right)-[E(Z)]^{2}}
$$

\subsection{PDIPM and GA}

AM is a problem of active power flow management, in other words a problem of OPF. This paper will use the Primal-Dual Interior Point Method (PDIPM) to solve the OPF problem. The decision variables include $P_{I D G, i}$, $Q_{I D G, i}, Q_{C i}, \operatorname{Tap}_{k}, P_{L c u r, i}$. The control variable $\boldsymbol{X}$, objective function and the Lagrange function are shown as below:

$$
\begin{aligned}
& \boldsymbol{X}=\left[\begin{array}{c}
\boldsymbol{\theta} \\
\boldsymbol{U} \\
\boldsymbol{P}_{g} \\
\boldsymbol{Q}_{g}
\end{array}\right] \\
& f(\boldsymbol{X})=\mathbf{1}_{n}^{T} \boldsymbol{P}_{I D G} \\
& L(\boldsymbol{X})=f(\boldsymbol{X})+\boldsymbol{\lambda}^{T} \boldsymbol{G}(\boldsymbol{X})+\boldsymbol{\tau}_{1}^{T}\left(\boldsymbol{H}_{1}(\boldsymbol{X})-\boldsymbol{s}_{1}\right)+ \\
& \boldsymbol{\tau}_{2}^{T}\left(\boldsymbol{H}_{2}(\boldsymbol{X})+\boldsymbol{s}_{2}\right)+\mu \sum\left(\ln s_{1 i}+\ln s_{2 i}\right)
\end{aligned}
$$

where $\boldsymbol{\theta}, \boldsymbol{U}$ are the bus voltage phase angle and amplitude vector; $\boldsymbol{P}_{\mathrm{g}}, \boldsymbol{Q}_{\mathrm{g}}$ are the active and reactive power vector of the generators, including the S/S (Substation), WTG and PVG; $f(\boldsymbol{X})$ is the objective function, $f(\boldsymbol{X}) \in \boldsymbol{R}^{1} ; \mathbf{1}_{n}^{T}$ is the row vector with all the elements $1 ; \boldsymbol{P}_{I D G}$ is the active power vector of IDG; $L(\boldsymbol{X})$ is the Lagrange function, $L(\boldsymbol{X}) \in \boldsymbol{R}^{1}$; $\boldsymbol{G}(\boldsymbol{X})$ is the equality constraint function, $\boldsymbol{G}(\boldsymbol{X}) \in \boldsymbol{R}^{l}, l$ is the number of the equality constraints; $\boldsymbol{H}_{1}(\boldsymbol{X}), \boldsymbol{H}_{2}(\boldsymbol{X})$ are the inequality upper limit and lower limit constraint function, $\boldsymbol{H}_{1}(\boldsymbol{X}) \in \boldsymbol{R}^{m}, m$ is the number of the inequality constraints; $\boldsymbol{s}_{1}, \boldsymbol{s}_{2}$ are the slack variables; $\lambda, \boldsymbol{\tau}_{1}, \boldsymbol{\tau}_{2}$ are the dual variable vectors; $\mu$ is the barrier parameter.

As the uncertainties are considered, the optimal tap positions suitable for all the uncertainties at time $t$ cannot be easily obtained by adding variables to PDIPM. So GA (genetic algorithm) [25-26] is used to obtain the best tap positions suitable for the uncertain circumstance at each time segment. The binary code is shown in Fig. 3.

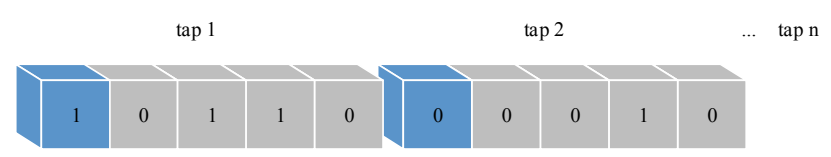

Fig. 3. The binary code of GA

Every chromosome has several taps, each tap consists of 5 binary bits as the tap range is $-8 \sim+8$. The first bit with blue color is the tap direction indication, 1 means adjusting upward, 0 means downward. The following 4 bits indicate the tap position. In Fig. 3 tap 1 position is +6 , tap 2 position is -2 . If the tap position limits are set to $0.95 \sim 1.05$ p.u., the total tap positions is 16 , the voltage of tap 1 is $1+(0.05 / 8) \times$ $6=1.0375$ p.u., the voltage of tap 2 is 0.9875 p.u.

\subsection{Solving methodology}

The OpenDSS (Open Distribution System Simulator) is a comprehensive electrical system simulation tool for electric utility distribution systems, which is implemented as in-process COM server DLL designed to be driven from a variety of existing software platforms [24]. As the data of IEEE radial distribution test feeders is available in the OpenDSS test cases, and openDSS can easily control the tap position of OLTC and VR. Furthermore openDSS has multi-period simulation mode, like daily mode, yearly mode, which can be used to deal with the multi-period optimization. We use OpenDSS to get the Y matrix for the PDIPM.

This paper uses Matlab to drive openDSS. The openDSS simulation mode is set with daily for the multi-period optimization. AM schemes are assumed to be independent in different hours. The uncertainties of loads, WTG and PVG are also independent. Different scenarios are selected for the IDG accommodation capability investigation under different active management schemes. The total procedure is shown in Fig.4:

The scenarios are defined as below: 


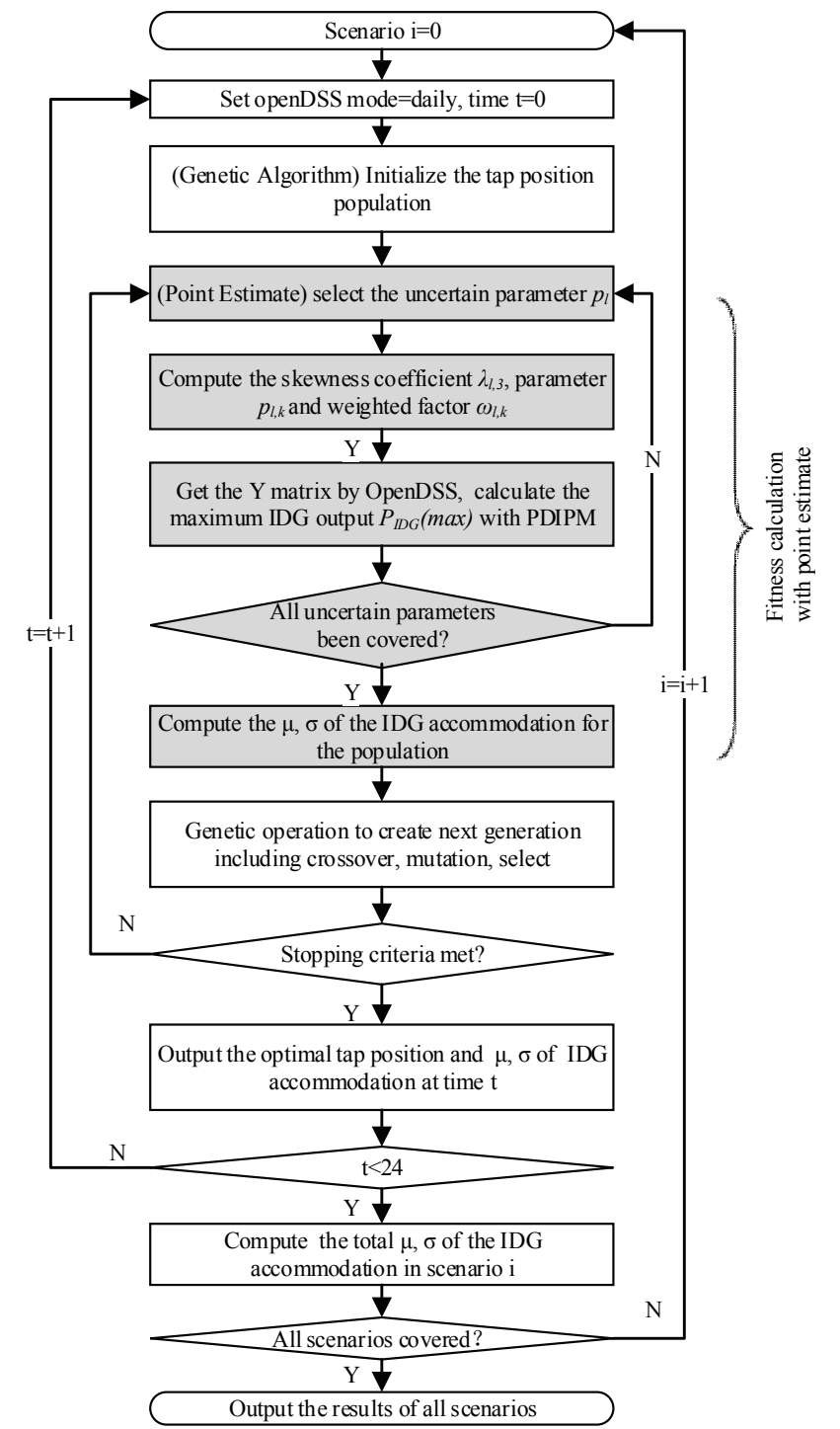

Fig. 4. The flow chart of the proposed IDG accommodation evaluation method

S1: IDGs, tap positions both fixed, if there is bus voltage or branch current violation, all the IDGs will be tripped; S2: IDGs are dispatchable, and the tap positions are fixed; S3: Both IDGs and the tap positions are dispatchable

\section{Results and Discussions}

The proposed model and method are tested on modified IEEE 123 node test feeders [29], which is a $4.16 \mathrm{kV}$ distribution networks. The structure and original installed elements of this feeder are shown in [29]. The uncertainties of the load are assumed to follow normal distribution, the mean value of the load is the basic load of the corresponding test feeder, and the standard deviation is assumed to be $10 \%$ of the basic load.

Normally, the long-time wind speed follows Weibull distribution; the illumination intensity follows Beta distribution [27-28]. The corresponding parameter of Weibull and Beta distribution is assumed. The cut-in wind speed, cut-out wind speed and rated speed are separately $3.8 \mathrm{~m} / \mathrm{s}, 24 \mathrm{~m} / \mathrm{s}$ and $12.8 \mathrm{~m} / \mathrm{s}$. The rated illumination intensity is $0.78 \mathrm{~kW} / \mathrm{m}^{2}$. The rated power of WTG and PVG is given in the cases.

The timing characteristic data of the daily load, wind speed forecasting, the illumination intensity forecasting data and the errors are assumed based on practical area. The illumination intensity at time 19:00-00:59, 01:00-4:59 is set to 0 as the nighttime. All the uncertainties of load, wind speed and illumination intensity are assumed in this paper. We can practically use the historical data of all the uncertainties, as we have used the point estimate probabilistic power flow.

Table 1. IEEE 123 node test feeder IDG installation information

\begin{tabular}{c|c|c|c|c}
\hline Node & Phase & DG Type & $P_{I D G} .(\mathrm{kW})$ & Power factor \\
\hline 56 & 3 & WTG & 300 & 0.95 \\
65 & 3 & WTG & 300 & 0.95 \\
76 & 3 & WTG & 300 & 0.95 \\
95 & 3 & PVG & 200 & 0.95 \\
100 & 3 & PVG & 200 & 0.95 \\
108 & 3 & PVG & 200 & 0.95 \\
\hline
\end{tabular}

The load, line and other information can be found in [29]. The IDG installation information is shown in Table 1. The tap adjusting range is $-8 \sim+8$, the tap position limits are set to $0.95 \sim 1.05$, that is every tap equals $0.00625 \mathrm{p}$.u. The HV source bus voltage is set to $1.05 \mathrm{p}$.u. The branch long-term normal capacity limitation is set to 500A.

The GA parameters used in this paper are as follows: population size $\mathrm{M}=50$, crossover probability $\mathrm{Pc}=0.9$, mutation probability $\mathrm{Pm}=0.1$. The tests are conducted on a Core(TM) i5-4200M CPU, 2.5GHz PC.

The installed IDGs are all 3-phase generators. The IDGs can be single phase configuration with a more targeted purpose for weak phases with lower voltage at the end of the feeder or overloaded phases. Table 2 gives out the IDG accommodation result over 24 hours and $\mathrm{C}$ phase voltage on bus 100 at time $t=12$. The $\mu$ is the total mean value of IDG accommodated over 24 hours, calculated with $\mathrm{E}\left(\mathrm{x}_{1}+\mathrm{x}_{2}+\ldots \mathrm{x}_{24}\right)=\mathrm{E}\left(\mathrm{x}_{1}\right)+\mathrm{E}\left(\mathrm{x}_{2}\right)+\ldots \mathrm{E}\left(\mathrm{x}_{24}\right)$, the $\sigma$ is the total standard deviation calculated with $\mathrm{D}\left(\mathrm{x}_{1}+\mathrm{x}_{2}+\ldots \mathrm{x}_{24}\right)=\mathrm{D}\left(\mathrm{x}_{1}\right)$ $+\mathrm{D}\left(\mathrm{x}_{2}\right)+\ldots \mathrm{D}\left(\mathrm{x}_{24}\right)$ with the assumption that $\mathrm{x}_{1}, \mathrm{x}_{2} \ldots, \mathrm{x}_{24}$ are independently and the covariance is zero. The voltage of scenario S3 is lower than S2 and S1, this is because in $\mathrm{S} 3$, the tap position adjusting lower the total voltage in order to accommodate more IDGs, while the IDG installed on bus 100 cannot draw back much. Also there is no linear relations between the installed IDG and the node voltage because of different tap positions in different IDG capacity installation. Compare scenario S3 with S2 in Table 2, we can see the tap adjusting can largely increase the IDG accommodation. This paper will focus on the decision variable $\operatorname{Tap}_{k}$, which is much more important for IDG 
Table 2. Mean and standard deviation results for the IEEE 123 node test feeder ( $E_{\text {IDG }}$ is the IDG accommodation over 24 hours, $\mathrm{V}_{100}$ is the $\mathrm{C}$ phase voltage at time $\mathrm{t}=12$ )

\begin{tabular}{c|c|c|c|c|c|c|c}
\hline \multirow{2}{*}{ Scenario } & \multicolumn{2}{|c|}{$\mathrm{S} 1$} & \multicolumn{2}{c|}{$\mathrm{S} 2$} & \multicolumn{2}{c}{$\mathrm{S} 3$} \\
\cline { 3 - 8 } \multicolumn{2}{c|}{} & $\begin{array}{c}\mathrm{E}_{\mathrm{IDG}} / \\
\mathrm{MWh}\end{array}$ & $\begin{array}{c}\mathrm{V}_{100} / \\
\text { p.u. }\end{array}$ & $\begin{array}{c}\mathrm{E}_{\mathrm{IDG}} / \\
\mathrm{MWh}\end{array}$ & $\begin{array}{c}\mathrm{V}_{100} / \\
\text { p.u. }\end{array}$ & $\begin{array}{c}\mathrm{E}_{\mathrm{IDG}} / \\
\mathrm{MWh}\end{array}$ & $\begin{array}{c}\mathrm{V}_{100} / \\
\text { p.u. }\end{array}$ \\
\hline \multirow{2}{*}{ IDG } & $\mu$ & 2.42 & 1.0071 & 14.76 & 1.0233 & 24.48 & 0.9946 \\
\cline { 2 - 8 } & $\sigma$ & 0.19 & 0.0089 & 1.11 & 0.0106 & 2.24 & 0.0113 \\
\hline 1.5 & $\mu$ & 0 & 1.0279 & 14.82 & 1.0288 & 36.54 & 0.9985 \\
\cline { 2 - 8 } IDG & $\sigma$ & 0 & 0.0062 & 1.20 & 0.091 & 3.28 & 0.0108 \\
\hline 2 & $\mu$ & 0 & 1.0280 & 14.80 & 1.0291 & 48.67 & 0.9973 \\
\cline { 2 - 8 } IDG & $\sigma$ & 0 & 0.0059 & 1.17 & 0.0102 & 5.36 & 0.0098 \\
\hline
\end{tabular}

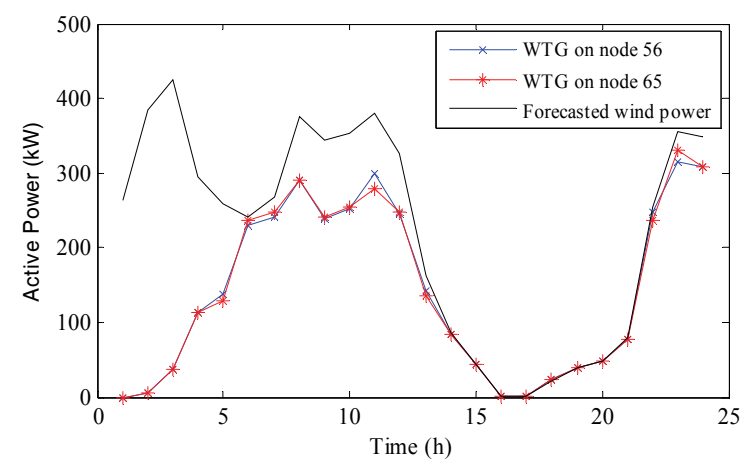

Fig. 5. WTG active power on node 56 and 65 at scenario S2, 2 IDG.

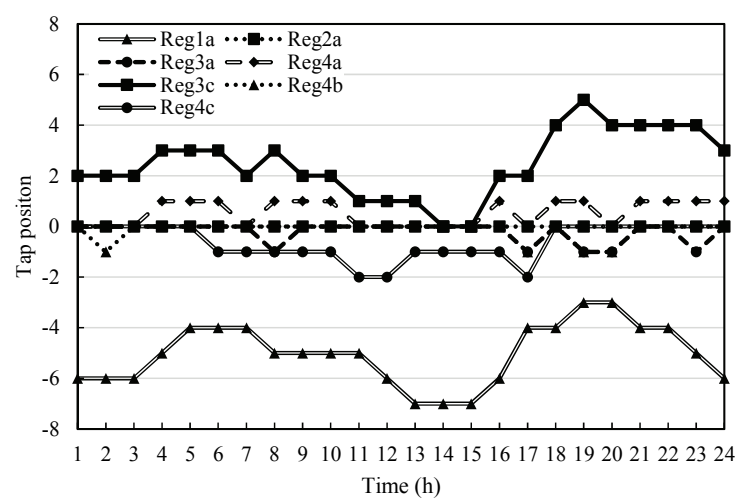

Fig. 6. Tap position over the day at scenario S3, 2 IDG.

accommodation.

Fig. 5 is the active power of WTG on node 56 and 65 with 2 IDG at scenario S2. We can see that without tap adjusting, the WTG accommodation are restricted by the network security constraints, especially at the time with high IDG output and lower load. The IDG curtailments for this two IDG are $3.88 \mathrm{MWh}$.

Fig. 6 is the tap position over the day with 2 IDG at scenario S3. Regla is the tap position indication of controllable VR between bus 150 and 149. Reg2a is the tap position indication of VR between bus 9 and 14. Reg3a, 3c are the tap position indications VR between bus 25 and 26. Reg4a, 4b, 4c are the tap position indications of VR between bus 160 and 67 . We can see the tap positions have not reached the limitation, there is still surplus for additional IDG penetration if we only consider the mentioned constraints in the model of section 3. The tap position of Reg1a is set lower for countering the effect of higher penetration of IDG, especially at 13:00 15:00 with peak load. But the much lower tap position in the substation will make the end bus without IDG installation voltage violation. Thus some VR like Reg3c is set higher a bit to lift the voltage of end bus.

Fig. 7 shows the Probability Density Function(PDF) and Cumulative Distribution Function(CDF) of Bus 100 $\mathrm{C}$ phase voltage probability distribution at time $\mathrm{t}=12$, scenario S3, 1.5IDG. It is obtained with Gram-Charlier expansion [21, 22]. We can see the point estimate method can effectively simulate the uncertainties. From Fig. 7(a), the AM coordination can decrease the system risks during distribution network operation, ensure the node voltage and branch current within the limits. The voltages of all the estimated points locate between $0.965 \sim 1.025$ from Fig. 7(b).

Fig. 8 shows the test feeder voltage profile at time $\mathrm{t}=12$, scenario $\mathrm{S} 3,1.5 \mathrm{IDG}$. The AM schemes including the DG output curtailment, reactive power compensation and the OLTC tap adjusting are known with the previous mentioned mathematics model and methodology. Fig. 8(a) is the voltage profile with all the tap positions manually fixed to 0 . We can see the IDG installations raise the total

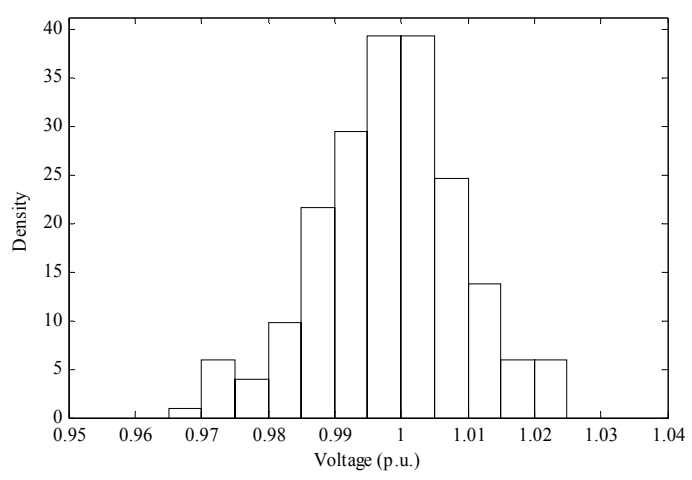

(a)

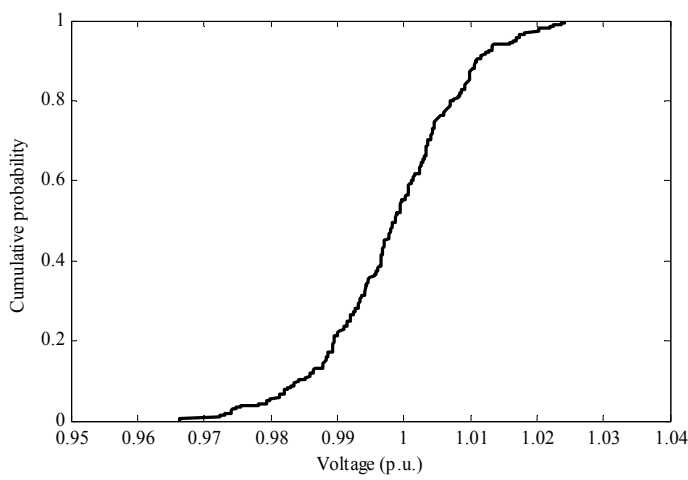

(b)

Fig. 7. Bus $100 \mathrm{C}$ phase voltage probability distribution at time $\mathrm{t}=12$, scenario S3, 1.5IDG: (a) PDF; (b) CDF 


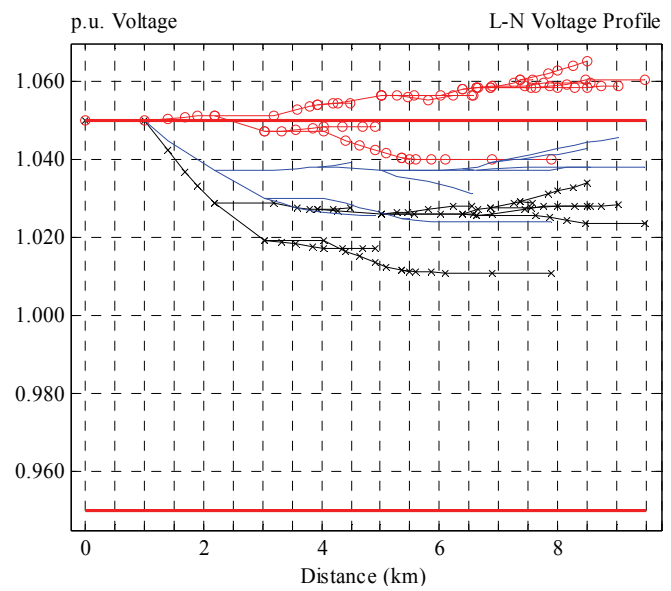

(a)

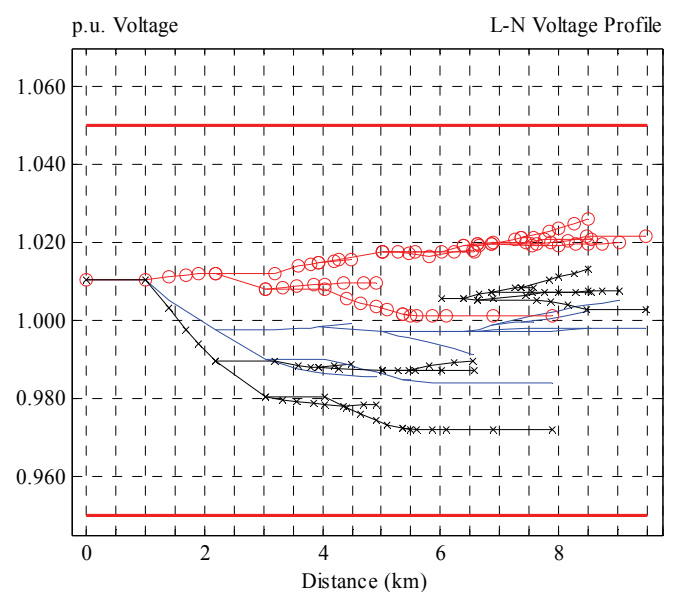

(b)

Fig. 8. IEEE 123 node test feeder voltage profile with peak load $\mathrm{t}=12$, scenario S3, 1.5IDG: (a) without tap adjusting, all tap positions are set to 0 ; (b) with the optimal tap positions [Reg1a Reg2a Reg3a Reg4a Reg3c Reg4b Reg4c] $=\left[\begin{array}{lllllll}-6 & 0 & 0 & 1 & 3 & 0 & 0\end{array}\right]$ (The bold horizontal red lines in the upper and lower side are the voltage limits. The red lines marked with circles are the $\mathrm{B}$ phase voltage distribution, the blue lines is the $\mathrm{C}$ phase voltage distribution, the black lines marked with crosses are the A phase voltage distribution.)

network voltage. Without tap adjusting, the IDG leads the violation of $B$ phase voltage, as the total loads of B phase are the lowest. Fig. 8(b) is the voltage profile with optimal tap positions, the proper tap adjusting can avoid the violation, improve the IDG accommodation. The voltage stepping on line 150-149(a phase), 16-67(a phase), 25-26(c phase) is due to the tap adjusting.

\section{Conclusion}

This paper analyzes the multiple AM schemes in ADN, proposes IDG optimal coordination operation model to increase the IDG accommodation based on the load and IDG timing characteristics and uncertainties. The scenario analysis method is used to analyze the IDG accommodation capability under different AM schemes. The proposed model and method are validated with modified IEEE 123 case. The conclusions are given below:

1) From the AM analysis, the improper interconnecting of IDG will cause the increasing of bus voltage, affect the safe and stable operation of distribution system. AM can improve the operation condition, increase the amount of IDG accommodated;

2) The multi-period IDG accommodation model considering uncertainties can completely and accurately evaluate the distribution network IDG accommodation capability. The application of AM can largely decrease the IDG curtailment during the operation. If there are only the bus voltage and branch capacity constraints considered, there is still surplus for additional IDG penetration in the cases mentioned in this paper.

\section{Acknowledgements}

This work was supported in part by the National High Technology Research and Development Program (863 Program) of China under Grant 2014AA051901 and National Natural Science Foundation of China under Grant 51261130473.

\section{References}

[1] International Energy Agency (IEA), World Energy Outlook 2010, [Online], Available: http://www.worldenergyoutlook.org/.

[2] IEA Statistics (2012). CO2 Emissions from Combustion-Highlights, [Online], Available: http://www.iea.org/statistics/.

[3] Ministry of Industry and Information Technology of the People's Republic of China, [Online], Available: http://www.miit.gov.cn/.

[4] State Grid Corporation of China, [Online], Available: http://www.sgcc. com.cn/.

[5] Ochoa L F. and Harrison G P, "Minimizing energy losses: Optimal accommodation and smart operation of renewable distributed generation", IEEE Trans. Power Systems, vol. 26 no. 1, pp. 198-205, Feb. 2011.

[6] Ochoa L F, Dent C J and Harrison G P, "Maximisation of intermittent distributed generation in active net-works", CIRED Seminar, 2008.

[7] Barton J P and Infield D G, "Energy storage and its use with intermittent renewable energy", IEEE Trans. Energy Conversion, vol. 19 no. 2, pp. 441-448, Jun. 2004.

[8] Fan Mingtian, Zhang Zuping, Su Aoxue, et al, 
"Enabling technologies for active distribution systems", Proceedings of the CSEE, vol. 33, no. 22, pp. 12-18, Aug. 2013.

[9] You Yi, Liu Dong, Yu Wenpeng, et al, "Technology and its trends of active distribution network", Automation of Electric Power Systems, vol. 36, no. 18, pp. 10-16, Sep. 2012.

[10] Chowdhury S and Crossley P, "Microgrids and active distribution networks", The Institution of Engineering and Technology, 2009.

[11] McDonald J, "Adaptive intelligent power systems: Active distribution networks", Energy Policy, vol. 36, no.12, pp. 4346-4351, 2008.

[12] Ault G, Currie R and McDonald J, "Active power flow management solutions for maximising DG connection capacity", IEEE Conference in Power Engineering Society General Meeting, 2006, pp. 1-5.

[13] Currie R, Ault G, Foote C, et al, "Active power-flow management utilising operating margins for the increased connection of distributed generation", IET Generation Transmission \& Distribution, vol. 1, no. 1, pp. 197-202, Jan. 2007.

[14] V. Calderaro, G. Conio, V. Galdi, G. Massa, et al, "Active management of renewable energy sources for maximizing power production", International Journal of Electrical Power \& Energy Systems, vol. 57, pp. 64-72, May 2014.

[15] Currie R, Ault G, McDonald J, "Methodology for determination of economic connection capacity for renewable generator connections to distribution networks optimised by active power flow management", IEE Proceedings in Generation Transmission and Distribution, vol. 153, no. 4, pp. 456-462, Jul. 2006.

[16] Siano P, Chen P, Chen Z, et al, "Evaluating maximum wind energy exploitation in active distribution networks", IET Generation Transmission \& Distribution, vol. 4, no. 5, pp. 598-608, May 2010.

[17] Gabash A and Li P, "Active-reactive optimal power flow in distribution networks with embedded generation and battery storage", IEEE Trans. Power Systems, vol. 27, no. 4, pp. 2026-2035, Nov. 2012.

[18] Dolan M J, Davidson E M, Kockar I, et al, "Reducing distributed generator curtailment through active power flow management", IEEE Trans. Smart Grid, vol. 5, no. 1, pp. 149-157, Jan. 2014.

[19] Simon Gill, Ivana Kockar and Graham W Ault, "Dynamic optimal power flow for active distribution network", IEEE Trans. Smart Grid, vol. 29, no. 1, pp. 121-131, Jan. 2014.

[20] C. D' Adamo, C. Abbey and S. Jupe, “Development and operation of active distribution networks: results of CIGRE C6.11 working group", Proceedings 21th International Conference on Electricity Distribution, Frankfurt, Jun. 2011.

[21] C.-L. Su, "Probabilistic load-flow computation using point estimate method", IEEE Trans. Power Systems, vol. 20, no. 4, pp. 1843-1851, Nov. 2005.

[22] J. M. Morales and J. Perez-Ruiz, "Point estimate schemes to solve the probabilistic power flow", IEEE Trans. Power Systems, vol. 22, no. 4, pp. 1594-1601, Nov. 2007.

[23] Hong H, "An efficient point estimate method for probabilistic analysis", Reliability Engineering \& System Safety, vol. 59, no. 3, pp. 261-267, Mar. 1998

[24] R. C. Dugan, "Reference Guide: The Open Distribution System Simulator (OpenDSS)", Electric Power Research Institute, Inc, 2012.

[25] B. Enacheanu, B. Raison, R. Caire, et al, "Radial network reconfiguration using genetic algorithm based on the matroid theory", IEEE Trans. Power Systems, vol. 23, no. 1, pp. 186-195, Feb. 2008.

[26] E. Carrano, R. Cardoso, R. Takahashi, et al, "Power distribution network expansion scheduling using dynamic programming genetic algorithm", IET Generation Transmission \& Distribution, vol. 2, no. 3, pp. 444-455, Apr. 2008.

[27] AE Feijoo, J Cidras and J.L.G Dornelas, "Wind speed simulation in wind farms for steady-state security assessment of electrical power systems", IEEE Trans. Energy Conversion, vol. 14, no. 4, pp. 1582-1588, Dec. 1999.

[28] MKC Marwali, M Haili, S.M Shahidehpour, et al, "Short term generation scheduling in photovoltaicutility grid with battery storage", IEEE Trans. Power Systems, vol. 13, no. 3, pp. 1057-1062, Aug. 1998.

[29] Kersting W H, "Radial distribution test feeders", IEEE Conference in Power Engineering Society Winter Meeting, 2001, 908-912.

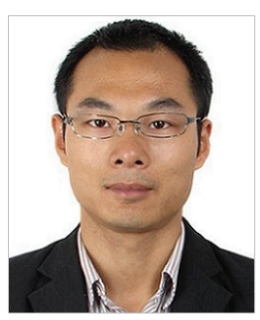

Haijun Xing He received his B.S. and M.S. degrees in Department of Electrical Engineering from Harbin Institute of Technology, Harbin, China, in 2002 and 2004 respectively. $\mathrm{He}$ is now purchasing his Ph.D. degrees in electrical engineering from Shanghai Jiao Tong University, Shanghai, China. His research interests include distribution network operation and planning.

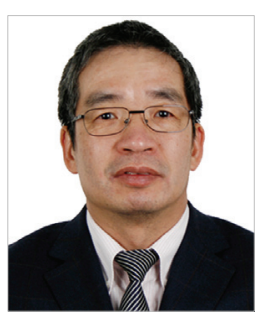

Haozhong Cheng He received his B.S., M.S., and Ph.D. degrees in power systems from Shanghai Jiao Tong University, Shanghai, China, in 1983, 1985, and 1998, respectively. $\mathrm{He}$ is now a Professor of Shanghai Jiao Tong University. His special fields of interest cover power system planning, voltage stability, harmonics, operation, and deregulation. 
Yi Zhang He received his B.S. and Ph.D. degrees in power systems from Sichuan University, Chengdu, China, in 2007 and 2012, respectively. He is now a Senior Engineer of Fujian Electric Power Research Institute. His special fields of interest cover power quality, active distribution network, and operational risk assessment. 